\title{
THE EFFECTS OF USING STROBOSCOPIC TRAINING ON SPORTS PERFORMANCE
}

\author{
Antonia Ioana TRIFU (VASILE) ${ }^{1 *}$, Monica STĂNESCU ${ }^{1}$ \\ ${ }^{1}$ National University of Physical Education and Sport, Faculty of Physical Education and Sport, Bucharest, \\ Romania \\ *Corresponding author: antoniatrifu@yahoo.com
}

https://doi.org/10.35189/dpeskj.2021.60.2.4

\begin{abstract}
Stroboscopic training is a form of athletic training during which the individual performs a motor task under intermittently opaque visual conditions. The purpose of this training is to enhance subsequent performance under normal visual conditions as a result of shifting attentional focus within the multisensory integration process. The training performed with the help of this technology that uses specially designed glasses increases the contribution of other somatosensory afferents to motor control by refocusing the individual's attention on proprioceptive feedback. Other benefits of stroboscopic training include improved short-term visual memory, improved capacity for anticipation as well as increased ability to focus in general but particularly on the central visual field and thus produce coherent movements. The current paper is a metaanalysis of the literature on stroboscopic training and its benefits for professional sport. In sports using stroboscopic (strobe) glasses, for example in hockey, basketball, football, volleyball, baseball or tennis, positive effects on some skills have been identified and training and testing protocols have been described. The aim of this paper is to highlight some practical and methodological benchmarks resulting from the use of stroboscopic training in various sports, and then transfer them to climbing training in order to enhance performance in this sport.
\end{abstract}

Keywords: strobe glasses, multisensory integration, cognitive training, sensory refocusing, visual memory.

\section{Introduction}

Increasing human performance in general and sports performance in particular is a constant concern of researchers interested in identifying new ways to integrate technology into the training specific to various sports.

Stroboscopic training is a form of athletic training during which the individual performs a motor task under intermittently opaque visual conditions. For this purpose, stroboscopic (strobe) glasses are used as part of modern technology designed to contribute to sensory refocusing within the multisensory integration process (Kim et al., 2017) but also to allow visual-cognitive training. Shifting visual perception in an intermittent, repeated and rapid way forces athletes to use more efficiently the limited visual information they receive and/or to consciously use additional analysers (such as kinaesthetic or auditory ones) in order to keep their movements effective (Wilkins \& Appelbaum, 2019). When visual perception is disrupted, the central nervous system will potentiate the contribution of other somatosensory afferents to motor control as a result of refocusing the individual's attention on proprioceptive feedback.

The interest in using stroboscopic glasses in the field of sport has arisen due to the fact that, at a high-performance level, external focus is superior to internal focus (Wulf, 2013), and shifting attention to the internal, proprioceptive sense contributes to an increase in 
performance. On the other hand, performing an exercise of increased difficulty during stroboscopic training changes an individual's subjective perception of physical exertion. Under normal conditions, which means without stroboscopic eyewear, the effort will seem easier, and moving objects will seem to move slower or faster (Smith \& Mitroff, 2012).

The current paper is a meta-analysis of the literature on stroboscopic training and its benefits for sports performance. The aim is to highlight the concrete ways of using this type of training in various sports (hockey, basketball, football, volleyball, baseball, tennis, badminton), with an emphasis on the achieved results and the training and testing protocols used by other researchers. Subsequently, the results can be transferred to the training specific to other sports.

\section{Topic Addressed}

\section{Stroboscopic technology}

Strobe glasses consist of liquid crystal lenses that will flicker to block light signals entering the visual system. Lenses alternate between opacity and transparency over predefined periods of time, reducing information and forcing individuals to process visual stimuli more efficiently.

Glasses can generate eight levels of alternation between transparent and opaque states, which differ in speed and difficulty. The slower the lens or lenses flicker, the harder the task becomes. Lenses can also be adjusted in three operating modes: A (both lenses flicker), B (left lens flickers, right lens is opaque), $\mathrm{C}$ (right lens flickers, left lens is opaque).

There are several types of stroboscopic glasses. Senaptec LLC (Senaptec Strobe) glasses have been developed subsequent to the design of Nike SPARQ Vapor Strobe Glasses (2011) and are based on the same manufacturing style, but their advantage consists in using lenses able to create a semi-transparent state that almost entirely blocks the light. In addition, the Senaptec digital application can be used, which allows remote control of the glasses via Bluetooth connection. (Senaptec, 2020b)

The Senaptec application has developed 15 sensory training modules to increase human performance. The 15 fundamental skills improved by the exercises with these glasses are: eye-hand coordination, Go/No-Go (to train quick decision-making ability and swift movement), dynamic vision (to train peripheral vision awareness and rapid eye movement), perception training, response inhibition (to train rapid decision-making ability and muscle response), spatial memory, spatial sequence, multiple object tracking, depth perception, nearfar shift, visual search, tempo, shape cancellation, visual motor integration, split attention (Senaptec, 2020a). This application has designed training plans for sports such as baseball, basketball, football, hockey, volleyball or tennis.

\section{Physiological mechanisms used in stroboscopic training}

"Approximately 80 percent of the sensory input to the brain comes from the visual system and systems to process visual information" (Clark et al., 2020, p. e9). Achieving sports performance involves, along with sports skills, the ability of visual-spatial analysis consisting 
of visuomotor skills that require the processing of sensory information and optimal coordination between the occipital lobe and motor cortex. Stroboscopic glasses have a significant contribution to enhancing this type of coordination.

Goodale and Milner (1992) described two patterns of visual information processing with two pathways formed in the primary visual area: dorsal and ventral streams. The dorsal stream involves conscious perception (the "what" component) of the characteristics of objects, long-term memory and action planning based on the recalled information. The ventral stream controls real-time orientation, prompt action and reaction to a visual stimulus (the "how" component). The authors explain that the ventral stream allows viewing the object, while the dorsal stream is responsible for spatial vision. These streams and their coordination with motor cortex are critical for optimal athletic performance. It is obvious that competitive performance puts emphasis on the reaction to visual stimuli, but conscious perception of the patterns to be recognised from the visual information are equally important for athletes.

In sport, visual perception depends on peripheral vision, dynamic visual acuity and visuomotor skills. In addition, it depends on complex cognitive functions. Sports performance is based on depth perception, spatial information processing, quick reaction to visual stimuli and the decision-making ability. Sensory information reaches the visual system via the retina and optic nerve and is processed in the visual pathways of the occipital lobe. Basically, the essence of visual training becomes the coordination between the information coming through the visual pathway and the upper cortical processing.

Jeong et al. (2020) have concluded in their study that athletes with poor visual stimulation (as a result of stroboscopic vision) tend to have a low level of dynamic postural control when performing a motor task in the lateral and medial directions (to the left and to the right). They also show greater confidence in visual information when somatosensory stimuli are lateral.

Multisensory integration of vestibular, visual and somatosensory afferents supports human movement through feedback mechanisms (Schmidt \& Lee, 2013). The sensations provided by the three analysers produce an internal pattern for recognising body position and movement in relation to the external environment (Asslander \& Peterka, 2014).

From another perspective, studies have shown that the risk of injury and fall during motor tasks is associated with sensorimotor imbalances, but there is no clear report on the information from each somatosensory afferent; one explanation would be excessive reliance on visual information (Pasma et al., 2015).

Mickelson et al. (2020) analysed gait speed and balance during tandem walking (in a straight line, with contact between the heel and the tip of the opposite foot) under conditions of impaired vision (due to strobe glasses) in different age groups (examining a child, a young person and an adult) and reached the following conclusions:

- as visual restriction increased, shoulder and pelvis movement speeds increased;

- comparisons between participants indicated that age affected performance, suggesting that younger people would rely more on visual sensory information for gait, while adults would rely more on other sensory stimuli such as vestibular senses and proprioceptors;

- the child had greater variability in gait speed and shoulder tilt, while the adolescent showed more variability in pelvic tilt; 
- the adult showed the greatest variability in shoulder tilt.

\section{The effects of stroboscopic training on different sports}

The use of stroboscopic training in sport was based on a series of scientific data that confirmed the possibility of increasing visual performance by carrying out specific tasks under controlled conditions. The premise of stroboscopic training is that it develops visualcognitive processes, which facilitates the ability to cope with suboptimal available information. It has been demonstrated that the following processes can be positively transferred when normal vision is restored: short-term visual memory (Appelbaum et al., 2012), anticipatory timing (Smith \& Mitroff, 2012), movement coherence and attentional focus on the central visual field (fovea centralis) (Appelbaum et al., 2011).

The effects of stroboscopic training are different depending on the specificity of each sport.

In cricket, Hopwood et al. (2011) conducted a study of 12 professional players who were asked to complete a video-based decision-making test and an on-field test before and after a 6-week training programme. Seven of them performed three additional visual-cognitive training sessions per week. In the post-test, these players showed significant differences in decision accuracy within the video-based test and improvements in their on-field performance as compared to the control group.

In baseball, Classé et al. (1997) determined visual reaction time for 213 athletes and pointed out that there was a strong relationship between visual reaction time and batting efficiency.

In football, McLeod (1991) examined a women's team and revealed that, after 4 weeks of visual training, they had improved their results in eye-hand, eye-foot and balance coordination tasks (Dribble Test) as compared to the control group. Another football study (Clark et al., 2015) showed the effects of visual training on decreasing concussion incidence in this sport.

Based on promising results regarding the enhancement of visual skills, stroboscopic training has been designed and used in various sports (American football, baseball, basketball, ice hockey, rugby, football) in order to test its effectiveness on motor performance.

In an experiment using stroboscopic training, Dynavision D2 interactive light board and pre-competition ball tracking tasks, Clark et al. (2020) demonstrated a reduction in injuries among athletes in the experimental group compared to the control group. These results were based on the improvement of athletes' awareness of the workspace (playing field) and the development of vigilance towards the prevention of injuries. At the same time, their incidence decreased from one year to another but also before and after a visual training session. Basically, the authors demonstrated and explained a decrease from 5.1 cases per 100 players between 2006 and 2010 to 1.4 cases per 100 players between 2010 and 2013.

This type of training improves neuro-visual processing, so athletes can optimally process on-field visual information, including peripheral vision information in order to react faster to environmental changes and therefore to possible injuries. Mental processes such as reaction time, visual processing and visual field are very important for injury prevention. Visual 
training also improves athletic performance as a result of a significant increase in eye-hand coordination ability, reaction time and vigilance on the playing field.

In hockey, Schwab and Memert (2012) show that visual training leads to an improvement in the control and motor activity of extraocular and intraocular muscles. The gaze becomes more focused on a point and helps the brain to process information from central vision but also peripheral vision. Another hockey study (Poltavski \& Biberdorf, 2015) demonstrated that $69 \%$ of variance in the goals scored by forwards could be predicted by the players' reaction time to a visual stimulus but also by their better visual memory, better visual discrimination and faster ability to shift focus from near to far objects (visual perception measurements). Another study of elite hockey players (Mitroff et al., 2013) showed that athletes improved their shooting accuracy by $18 \%$. However, the results of this study cannot be extrapolated to larger populations due to the small group of research participants ( 6 in the experimental group and 5 in the control group).

In volleyball, Kroll et al. (2020) conducted a study of 13 NCAA Division I female athletes who had to complete a testing protocol consisting of $0.38-\mathrm{m}$ depth jumps under conditions of full vision and stroboscopic vision at low $(1.75 \mathrm{~Hz})$ and medium $(4 \mathrm{~Hz})$ frequencies. The researchers showed that stroboscopic vision had an influence on the depth-jump values (measured by jumping force and vertical reaction force) and the biomechanics of exercise due to sensorimotor overload. Therefore, stroboscopic training can be used as a strategy to change the intensity of plyometric movements by contrast to traditional mechanical overload exercises.

In tennis, Schootemeijer and Visch (2017) highlighted that the hitting accuracy of elite players in the Dutch National U-16 Team had significantly improved after stroboscopic training as compared to the control group.

A pilot study (Wilkins et al., 2017) of three elite youth football goalkeepers used semistructured interviews and pointed out three main beliefs related to the benefits of stroboscopic training. Thus, the surveyed athletes felt that: it had improved their visual and perceptual skills (focus, reactions and judgement); it had improved their on-field goalkeeping performance; it was both effortful and enjoyable.

Another study (Ballester et al., 2017) conducted with 20 non-athlete individuals compared coincidence-anticipation performance during normal vision versus stroboscopic vision. Participants were required to estimate the arrival time of a real object moving with constant acceleration. They made several errors (accuracy and variability) in normal vision conditions as a function of time-on-task but maintained their performance in stroboscopic vision conditions. The authors concluded that participants failed to have good performance in normal vision conditions due to monotony and attentional underload. Instead, stroboscopic training placed a higher demand on their visual-spatial memory for movement extrapolation and thus they did not experience the typical decrease in vigilance and attention while performing the task. These results suggest promising short-term adaptation effects from stroboscopic training, but further research should consider for how long participants can maintain attention during physical exertion and whether there are negative effects of this type of training when normal vision is restored. 
Practical and methodological benchmarks for using stroboscopic training in various sports

So far, no unique protocol has been found in the literature for the exact duration in which athletes work wearing strobe glasses, because each study has designed its own training protocol (Wilkins \& Appelbaum, 2019), depending on the sport. Thus, the identified protocols last from 5-7 minutes (Smith \& Mitroff, 2012) to 635 minutes (Wilkins \& Gray, 2015).

Wilkins and Appelbaum (2019) conducted an analysis of training protocols in the literature and highlighted the duration of training, the number of sessions, the motor tasks to be performed and the frequency at which stroboscopic glasses were set.

In the study by Appelbaum et al. (2011), 2-10 sessions of 20-30 minutes each were scheduled, which included motor tasks designed by the strobe application and motor tasks specific to the game of football (passing and dribbling). The frequency level of strobe glasses was in the range of 1-6 Hz.

In another study by Appelbaum et al. (2012), the same frequency level (1-6 Hz) was used during 2-7 sessions of 15-45 minutes as well as application-designed tasks and ball passing.

In the study by Smith and Mitroff (2012), participants worked with strobe glasses for one minute at a frequency of $4 \mathrm{~Hz}$ throughout 5-7 sessions.

Mitroff et al. (2013) scheduled 16 sessions of at least 10 minutes each, with players performing hockey-specific motor tasks (skating, passing) but also non-specific activities (to develop balance and coordination skills), and the frequencies of strobe glasses ranged from 1 to $6 \mathrm{~Hz}$.

Wilkins and Gray (2015) developed a 165-minute protocol for motor activities (with a tennis ball) in which athletes wore strobe glasses set to a frequency ranging between 1 and 6 $\mathrm{Hz}$, using the tasks designed by the Nike application.

Subsequently, Wilkins et al. (2017) developed a 635-minute football protocol with strobe glasses set to 1-6 Hz for tasks that involved catching the tennis ball and specific tasks for the goalkeeper.

In the study by Hülsdünker et al. (2018), athletes performed 12-20 sessions of 12-15 minutes each with strobe glasses set to a frequency ranging from 5 to $6 \mathrm{~Hz}$, using badmintonspecific protocols. The authors showed positive effects of stroboscopic training on motor performance in a target group of 10 elite badminton athletes ( 5 in the experimental group who worked with strobe glasses and 5 in the control group). The researchers conclude that stroboscopic training may be more effective than conventional visuomotor training even in high-level athletes.

\section{Applications of stroboscopic training to climbing}

The benefits of stroboscopic training can also be transferred to other sports such as climbing. Although less used at present, it opens up new possibilities for investigating and improving the training of climbers, orienting research towards the verification of the following hypotheses: 
- the tactical training of climbers and implicitly their tactical thinking can be enhanced as a result of analysing the information from kinaesthetic stimuli and focusing on the processing of information from grips;

- internal focus on the kinaesthetic analyser leads to increased body awareness, with an effect on the efficiency of movements that a climber has to perform.

A hypothesis that supports the applicability of this type of training to climbing is the increase of central visual acuity with an effect on the efficient calibration of the grip. If peripheral vision is very important in ball sports because it helps to observe in advance and as quickly as possible the movement of the ball coming towards the player, it becomes essential in climbing where it helps the athlete to perform the grip in the best grasping area. Tactical decision involves adjusting the depth at which the grip will be performed depending on its type.

Blasing et al. (2014) explain that experienced climbers (compared to beginners) choose more purposefully which characteristics of a climbing hold are more relevant for their task. The visual analyser must send information to the cortex regarding the length, width and shape of the holds so that it can process which is the best place to grasp and the position of the whole body that will allow for the grip to be performed as efficiently as possible.

Another argument in favour of applying this type of training to climbing refers to its effect on increasing both focused attention and divided attention. On the one hand, a correlation can be made between the level of divided attention and the number of holds along the route. It often happens that athletes do not focus their attention on all the holds existing on the climbing wall, especially those for the feet, and therefore do not make an efficient movement or even fail to complete the route because "they have not seen the foothold".

Thus, we propose the implementation of Miller's concept of number, which is defined as the number of items that can be stored in the human mind and is theoretically limited (Linhares et al., 2011). In climbing, this number would mean the number of holds viewed and retained in the athlete's memory. As this number increases, the athlete uses all the kinaesthetic stimuli that the route offers, which leads to increased sports performance. This cognitive process is positively correlated with visual memory. These two cognitive processes are supplemented by efficient visualisation for completing the route. In climbing, visualisation is the process by which an athlete builds a mental picture of their own movements to the top.

In tactical terms, the literature (Hurni, 2003) focuses on the method of visualising the route in four planes: the first plane - for the arm movements, the second plane - for observing the footholds, the third plane - for objectifying places to secure the loops and shake the arms, and the fourth plane reunites the three previous ones.

In stroboscopic training, before climbing with eyewear, the athlete must achieve better visualisation in order not to waste time during the ascent looking for footholds, resting places, places to secure the loops or to decide how to grasp the holds.

Lack of visual stimulus forces athletes to start with an ascent plan from which any deviation means loss of energy because of both physical exertion and difficult vision. Thus, on the next ascent that would be done without glasses, the climbing efficiency will increase due to the use of all the kinaesthetic and visual stimuli, without losing any of them, and proper visualisation. 
Improving the decision-making ability, as demonstrated in the literature, is also beneficial for the tactical thinking of bouldering athletes. Increased decisional ability is needed to make decisions in approaching the route. The climbing wall offers several planes to perform the ascent using different techniques, but the athlete must pick one and not deviate or deviate as little as possible from it so as to save energy and avoid lactate accumulation in the muscles.

Last but not least, the most important argument that supports the applicability of stroboscopic training to climbing is the sensory modification that helps increase the importance of kinaesthetic stimuli to the detriment of visual ones. It is known that what differentiates climbing from other sports in terms of factors that condition performance is the specialised kinaesthetic system (Trifu, 2020). Therefore, refocusing the athlete's attention on proprioceptive feedback can bring benefits to the climber's sports performance.

\section{Conclusion}

It is important to highlight that stroboscopic training allows training sessions to take place in natural contexts. Athletes can perform exercises specific to their sports in a familiar place, without needing to move to another game room, another field or a specialised room. However, the training must comply with a specific protocol that is different from the classic format of daily workouts, more precisely it has to be adjusted in terms of duration (per se or stroboscopic training), exercises, frequency of exercises with strobe glasses and flickering rate, all of this depending on each athlete's experience and the specificity of the sport.

Researchers working with stroboscopic equipment claim that a post-test immediately after training can best objectify the training effects, but it is fundamental to objectify for how long the post-training effects will persist. In addition, Gray (2017) emphasises the idea of objectifying the effects of transferring the skills learned through stroboscopic training to sports performance. Thus, we consider it important to measure visual, perceptual or cognitive skills but also the variables specific to sports performance ability by measuring specific motor skills.

According to the analysed studies, an effect of stroboscopic training would be sensory modification (by interrupting the visual stimulus) and improved processing of stimuli coming from other sensory pathways (such as the kinaesthetic or auditory analyser).

The effects of stroboscopic training on athletes' performance recommend it as a form of organising cognitive-motor training.

\section{References}

Appelbaum, L. G., Schroeder, J. E., Cain, M. S., \& Mitroff, S. R. (2011). Improved visual cognition through stroboscopic training. Frontiers in Psychology, 2: 276. https://doi.org/10.3389/fpsyg.2011.00276

Appelbaum, L. G., Cain, M. S., Schroeder, J. E., Darling, E. F., \& Mitroff, S. R. (2012). Stroboscopic visual training improves information encoding in short-term memory. Attention, Perception, \& Psychophysics, 74(8), 1681-1691.

https://doi.org/10.3758/s13414-012-0344-6 
Asslander, L., \& Peterka, R. J. (2014). Sensory reweighting dynamics in human postural control. Journal of Neurophysiology, 111(9), 1852-1864. https://doi.org/10.1152/jn.00669.2013

Ballester, R., Huertas, F., Uji, M., \& Bennet, S. J. (2017). Stroboscopic vision and sustained attention during coincidence-anticipation. Scientific Reports, 7(1): 17898. https://doi.org/10.1038/s41598-017-18092-5

Blasing, B. E., Guldenpenning, I., Koester, D., \& Schack, T. (2014). Expertise affects representation structure and categorial activation of grasp postures in climbing. Frontiers in Psychology, 5: 1008. https://doi.org/10.3389/fpsyg.2014.01008

Clark, J., Betz, B., Borders, L., Kuehn-Himmler, A., Hasselfeld, K., \& Divine, J. (2020). Vision training and reaction training for improving performance and reducing injury risk in athletes. Journal of Sports and Performance Vision, 2(1), e8-e16. https://doi.org/10.22374/jspv.v2i1.4

Clark, J. F., Graman, P., Ellis, J. K., Mangine, R. E., Rauch, J. T., Bixenmann, B., Divina, J., Colosimo, A. J., \& Myer, G. D. (2015). An exploratory study of the potential effects of vision training on concussion incidence in football. Optometry \& Visual Performance, $3(2), 116-125$.

Classé, J. G., Semes, L. P., Daum, K. M., Nowakowski, R., Alexander, L. J., Wisniewski, J., Beisel, J. A., Mann, K., Rutstein, R., Smith, M., \& Bartolucci, A. (1997). Association between visual reaction time and batting, fielding, and earned run averages among players of the Southern Baseball League. Journal of American Optometric Association, 68(1), 4349. PMID: 9037989

Gray, R. (2017). Transfer of training from virtual to real baseball batting. Frontiers in Psychology, 8: 2183. https://doi.org/10.3389/fpsyg.2017.02183

Goodale, M. A., \& Milner, A. D. (1992). Separate visual pathways for perception and action. Trends in Neuroscience, 15(1), 20-25. https://doi.org/10.1016/0166-2236(92)90344-8

Hopwood, M. J., Mann, D. L., Farrow, D., \& Nielsen, T. (2011). Does visual-perceptual training augment the fielding performance of skilled cricketers. International Journal of Sports Science \& Coaching, 6(4), 523-536. https://doi.org/10.1260/1747-9541.6.4.523

Hülsdünker, T., Rentz, C., Ruhnow, D., Käsbauer, H., Strueder, H. K., \& Mierau, A. (2018). The effect of a 4-week stroboscopic training on visual function and sport-specific visuomotor performance in top level badminton players. International Journal of Sports Physiology and Performance, 14(3), 343-350. https://doi.org/10.1123/ijspp.2018-0302

Hurni, M. (2003). Coaching climbing: A complete program for coaching youth climbing for high performance and safety. Guilford, Conn.: Falcon.

Jeong, H., Hopkins, T., Han, S., \& Lee, H. (2020). Stroboscopic vision-induced sensory reweighting during dynamic postural control. Medicine \& Science in Sports \& Exercise, 52(7S): 55. doi: 10.1249/01.mss.0000670612.73903.50

Kim, K.-M., Kim, J.-S., \& Grooms, D. R. (2017). Stroboscopic vision to induce sensory reweighting during postural control. Journal of Sport Rehabilitation, 26(5). https://doi.org/10.1123/jsr.2017-0035

Kroll, M., Preuss, J., Ness, B. M., Dolny, M. \& Louder, T. (2020). Effect of stroboscopic vision on depth jump performance in female NCAA Division I volleyball athletes. Sports Biomechanics, 1-11. https://doi.org/10.1080/14763141.2020.1773917

Linhares, A., Chada, D. M., \& Aranha, C. N. (2011). The emergence of Miller's magic number on a sparse distributed memory. PLoS ONE, 6(1): e15592. https://doi.org/10.1371/journal.pone.0015592

McLeod, B. (1991). Effects of eyerobics visual skills training on selected performance measures of female varsity soccer players. Perceptual and Motor Skills, 72(3), 863-866. https://doi.org/10.2466/pms.1991.72.3.863 
Mickelson, E., Schmit, K., \& Knakmuhs, K. (2020). Effects of stroboscopic vision on gait speed and balance during tandem walking. https://red.library.usd.edu/idea/4/

Mitroff, S. R., Friesen, P., Bennett, D., Yoo, H., \& Reichow, A. W. (2013). Enhancing ice hockey skills through stroboscopic visual training: A pilot study. Athletic Training \& Sports Health Care, 5(6), 261-264. https://doi.org/10.3928/19425864-20131030-02

Pasma, J. H., Engelhart, D., Maier, A. B., Schouten, A. C., van der Kooij, H., \& Meskers, C. G. (2015). Changes in sensory reweighting of proprioceptive information during standing balance with age and disease. Journal of Neurophysiology, 114(6), 3220-3233. https://doi.org/10.1152/jn.00414.2015

Poltavski, D., \& Biberdorf, D. (2015). The role of visual perception measures used in sports vision programmes in predicting actual game performance in Division I collegiate hockey players. Journal of Sports Science, 33(6), 597-608. https://doi.org/10.1080/02640414.2014.951952

Schmidt, R. A., \& Lee, T. D. (2013). Motor programs. In R. A. Schmidt \& T. D. Lee (Eds.), Motor learning and performance: From principles to application (pp. 89-122). Human Kinetics.

Schootemeijer, S., \& Visch, L. (2017). Effect of stroboscopic training on the groundstroke in skilled youth tennis players. Student Research Conference 2017, 3(160-163).

Schwab, S., \& Memmert, D. (2012). The impact of a sports vision training program in youth field hockey players. Journal of Sports Science \& Medicine, 11(4), 624-631.

PMID: 24150071

Senaptec. (2020a). Senaptec Sensory Station. https://www.senaptec.com/product/sensory-station

Senaptec. (2020b). Senaptec Strobe. https://www.senaptec.com/product/senaptec-strobe-standard

Smith, T. Q., \& Mitroff, S. R. (2012). Stroboscopic training enhances anticipatory timing. International Journal of Exercise Sciences, 5(4), 344-353. PMID: 27182391

Trifu, A. (2020). Funcționarea intrapsihică a sportivilor de performanță din escalada românească [The intrapsychic functioning of performance athletes in Romanian climbing] (Teză de disertație). UNEFS București.

Wilkins, L., \& Appelbaum, L. G. (2019). An early review of stroboscopic visual training: Insights, challenges and accomplishments to guide future studies. International Review of Sport and Exercise Psychology, 13(1), 65-80. https://doi.org/10.1080/1750984X.2019.1582081

Wilkins, L., \& Gray, R. (2015). Effects of stroboscopic visual training on visual attention, motion perception, and catching performance. Perceptual and Motor Skills, 121(1), 57-79. https://doi.org/10.2466/22.25.pms.121c11x0

Wilkins, L., Nelson, C., \& Tweddle, S. (2017). Stroboscopic visual training: A pilot study with three elite youth football goalkeepers. Journal of Cognitive Enhancement, 2(1), 3-11. https://doi.org/10.1007/S41465-017-0038-Z

Wulf, G. (2013). Attentional focus and motor learning: A review of 15 years. International Review of Sport and Exercise Psychology, 6(1), 77-104. https://doi.org/10.1080/1750984X.2012.723728 